\title{
Terrain Roughness Measurement from Elevation Maps
}

\author{
Regis Hoffman Eric Krotkov \\ School of Computer Science \\ Carnegie Mellon University \\ Pittsburgh, PA. 15213 U.S.A.
}

\begin{abstract}
The Autonomous Planetary Rover Project at Camegie Mellon University is investigating the use of geometric information obtained from terrain elevation maps for mobile robot planning and control. We review how surface geometry has been characterized by surface roughness parameters, and why several of these parameters must be combined to form a vector roughness measurement. Next we propose a technique to localize and extract the intrinsic roughness from terrain elevation maps, and show how this can be used to characterize terrain. Keywords: Terrain analysis, surface roughness, mobile robots.
\end{abstract}

\section{Introduction}

The Autonomous Planetary Rover Project at Carnegie Mellon University is developing and demonstrating an autonomous, legged mobile robot that can survive, explore, and sample in rugged, outdoor environments, such as those found on the surfaces of planets [1]. During its operation, the rover will use terrain elevation maps to plan unobstructed paths, select footfall locations, and choose areas of sampling interest [3,6]. Each of these tasks uses geometric properties of the planetary surface. For example, the terrain geometry is critical in mobile robot path planning, since a path over flat terrain is preferable to a path over terrain with many surface irregularities, and in sampling tasks, surface geometry may indicate regions of geological interest. The goal is to develop theories for characterizing surface geometry and explore their usefulness for planetary exploration robots.

Properties of the surface geometry are generally labeled surface roughness parameters. Previous work in the measurement and use of surface roughness comes from the fields of geology and geomorphology $[4,5,7]$ and surface metrology $[8,9,10]$. Geologic surface roughness is concerned with characterizing small scale terrain features ( $<3$ meters in height) for terrain typing and traversability determination. Surface metrology is the study of surface finish, especially that of machined surfaces. 

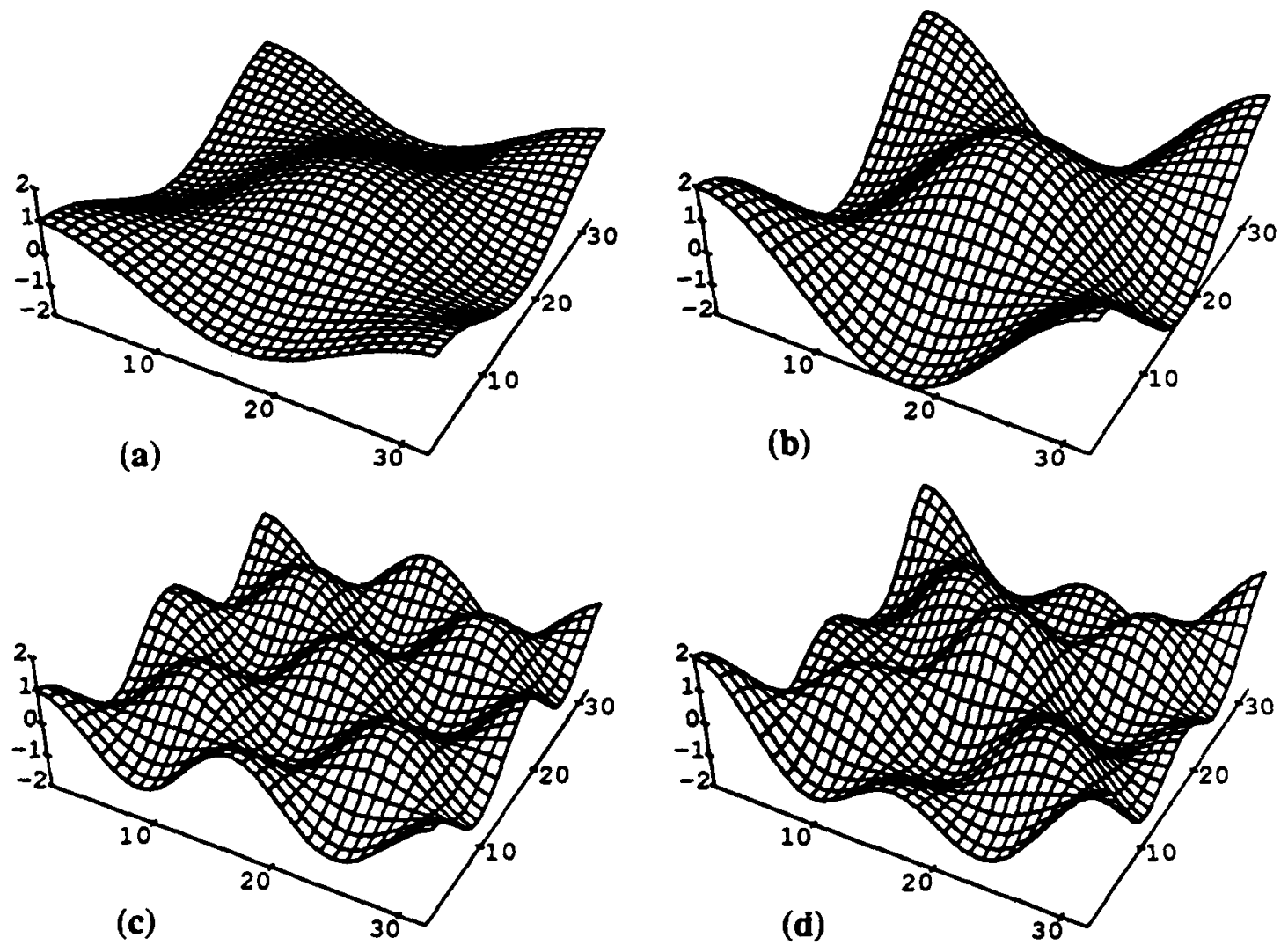

Figure 1: Terrain Surfaces

Most work with surface roughness analysis of terrain involves the use of a single parameter. We argue that a single parameter is insufficient to specify surface roughness and must be replaced by a vector quantity. A second shortcoming in surface roughness is that it is not an intrinsic property of the surface, but depends on the direction of measurement and surface orientation.

We present a vector measurement of terrain surface roughness that includes amplitude, frequency, and autocorrelation components, and is intrinsic to the surface. Terrain regions are then classified based on the roughness measurement.

\section{Terrain Roughness}

The concept of surface roughness is ambiguous. For example, consider the surfaces in figure 1. Intuitively, the surface in (b) is rougher than surface (a). More precisely, comparing two sinusoidal surfaces with the same frequency, the one with the larger amplitude would be considered rougher. Comparing surface (a) with (c), sinusoidal surfaces of the same amplitude but different 
frequencies, the one with the higher frequency is called rougher. Surface (d) exhibits irregularly spaced ridges - this is considered rougher than a surface of regularly spaced features. Thus the periodic repetition of prominent features must enter into the idea of roughness.

What are the desired qualities of a roughness measurement? We propose that any roughness measurement:

- Must discriminate between surfaces of different amplitudes, frequencies, and correlation.

- Be an intrinsic property of the surface, invariant with respect to rotation or translation.

- Be a local, not global measure of the surface.

- Have intuitive or physical meaning.

In the next section we review previous work on surface roughness and show that it does not meet the above criteria.

\section{Measures of Roughness}

Many different measures of roughness have been developed to parameterize machined surfaces (surface metrology) [2]. By considering examples of standard surface roughness parameters, it is shown that these violate the properties desired in an estimate of the roughness. The most important objection is the lack of discrimination in amplitude and frequency. Because very different surface profiles can yield identical roughness values, it is shown that a single quantity cannot adequately characterize the surface roughness.

One common measure of roughness is $R_{a}$ defined as the Center Line Average:

$$
R_{a}=\frac{1}{L} \int_{0}^{L}|y| d x
$$

This measure is not sensitive to amplitude differences as shown in the case comparing a DC signal with a square wave:

$$
\begin{gathered}
y_{1}(x)=y_{0} \quad 0 \leq x \leq 2 \\
y_{2}(x)=\left\{\begin{array}{rl}
y_{0} & 0 \leq x \leq 1 \\
-y_{0} & 1 \leq x \leq 2
\end{array}\right.
\end{gathered}
$$

In each case, the value of $R_{a}$ is $y_{0}$, even though the amplitude distributions are very different. Another possible measure of surface roughness is $R_{q}$ or the $R M S$ value:

$$
R_{q}=\left(\frac{1}{L} \int_{0}^{L} y^{2} d x\right)^{\frac{1}{2}}
$$


The case of two sinusoids of frequency $f_{1}$ and $f_{2}$ indicates that this measure is not a good frequency discriminator, as both sinusoids yield the same value of the roughness parameter $R_{q}$.

A more recent method of estimating surface roughness is to fit a plane to a surface, and use the error (or residuals) as an estimate of the surface roughness [11] (in the 1-D case this amounts to fitting a line to a set of points by a least squares technique). In the case of two sinusoidal surfaces of differing frequencies, plane fitting suffers from a fundamental shortcoming by producing the same roughness estimation. This can be understood by considering a sampled pure sinusoidal signal $x(n)$. In the 1-D case of fitting a line to this signal, the error in fit is:

$$
\text { Error }=\sum_{0}^{N-1}|x(n)|^{2}=\frac{1}{N} \sum_{0}^{N-1}|X(k)|^{2} \quad \text { Parseval's Theorem }
$$

For pure sine or cosine function with frequency $f_{c,}$ the spectrum is a pair of delta functions at $\pm f_{c}$ with magnitude $\frac{1}{2}$. The sum of the squares of the Fourier coefficients is $\frac{1}{2}$, independent of frequency. This idea extends to the 2-D case of a sinusoidal surface, and again the plane fit error is independent of the frequency. Since we argue that a sinusoidal surface of higher frequency is rougher than one of lower frequency, the plane fit error is not a good discriminator of surface roughness.

These arguments suggest that no single number can completely describe surface roughness, a set of numbers must be used. These numbers must discriminate between surfaces of different amplitudes, frequencies, and correlation.

Another deficiency is that these standard roughness measures are not intrinsic properties of the surface, but depend on the translation and orientation of the surface. The case of two surfaces described by $s(n)$ and $s(n)+C$ where $C$ is a constant, yields different values of $R_{a}$, even though the intrinsic roughness is identical. A final shortcoming is the lack of physical intuition in numbers such as the RMS value. For example, how does $R_{q}$ change as the terrain slopes increase?

A technique is next proposed that meets the desired qualities in a roughness measurement. Estimates of the local surface are made, and statistical parameters are extracted from Fourier transforms of surface.

\section{Roughness Estimation by Fourier Analysis}

A proposed method of estimating surface roughness using Fourier analysis by Stone and Dugundji [7] was used in studying geological surface irregularities measured from a fixed point of elevation that displayed internal differences of elevation from $7 \mathrm{~cm}$ to $3 \mathrm{~m}$ (microrelief features). This method measures roughness along specific directions of a surface and includes amplitude, frequency, and autocorrelation terms. 
This technique expresses roughness by six values obtained from the Fourier components of discretized terrain profiles (or slices). We selected three components that have the most physical meaning: ${ }^{1}$

$M$ The Simple Microrelief Factor, the expected range of heights of prominent microrelief features. The larger the value, the taller the microrelief features that can be expected.

$P$ The Variation in Slope of the Microrelief Packet, is the expected range of slopes of prominent microrelief features. The larger the value of $P$, the greater is the variance in the slope.

$K$ The Structural Similarity Factor indicates the tendency of the microrelief features to be repeated. The larger is $\mathrm{K}$, the more diverse are the microrelief, either in spacing or in form. $^{2}$

These values can be computed by taking expected values of the Fourier transform $X(n)$ of the terrain profile $x(n)$. These values are given by:

$$
\begin{aligned}
& M=\operatorname{Var}(x(n))=\sum_{0}^{N-1}|X(n)|^{2}-|X(0)|^{2} \\
& P=\operatorname{Var}(\dot{x}(n))=\sum_{0}^{N-1} f_{0}^{2} n^{2}|X(n)|^{2} \\
& K=1-\frac{\sum_{0}^{N-1}|X(n)|^{4}-|X(0)|^{4}}{\left(\sum_{0}^{N-2}|X(n)|^{2}-|X(0)|^{2}\right)^{2}}
\end{aligned}
$$

This choice of parameters has two advantages:

- They have physical meaning. A surface with large amplitudes will have an $M$ value greater than a surface with small amplitudes. $K$ is somewhat less intuitive, but for a signal of bandlimited white noise, $K \rightarrow 1$, and for a pure cosine signal, $K=0$.

- There is a consistent representation in the frequency domain.

This method does provide the necessary vector estimation of roughness, but does not measure the intrinsic surface roughness. We have extended this work by transforming the surface (to measure its intrinsic properties) and localizing it (for purposes of region classification).

\footnotetext{
'The other three components are essentially combinations of the three chosen, so no information is lost by selecting three.

${ }^{2}$ This $K$ is actually $1-K$ as defined by Stone. This was altered to have all components consistently indicate increasing roughness the larger the value of $M, P$ and $K$.
} 


\section{Estimating the Intrinsic Roughness}

A problem with Stone's technique is that it does not measure the intrinsic roughness of the surface; because it depends on the direction of measurement, it is influenced by the rotation and translation of the surface. For example, consider two terrain profiles of the same shape, but different height offset. Because there is a DC term in the calculation of the parameter $M$, the terrain profiles will have different roughness values. Ideally, the intrinsic roughness of the surface, independent of its orientation or translation, should be estimated.

The initial step is to estimate the underlying surface parameters. We assume that the measured input signal is the intrinsic surface rotated and translated by some transformation $T$. Given an input measured surface $s(n)$, the intrinsic surface $s_{i}(n)$ is estimated by the following procedure:

1. A least squares method fits a line $e^{3}$ to the signal $s(n)$. This line estimates the transformation $T$ that rotates and translates the intrinsic surface description $s_{i}(n)$ into $s(n)$ :

$$
s(n)=T s_{i}(n)
$$

2. The signal $s(n)$ is then transformed to remove the rotation and translation. More precisely:

$$
s_{i}(n)=T^{-1} s(n)
$$

Roughness estimates for $s_{i}(n)$ will now be estimates of the intrinsic properties of the surface.

\section{Localization of Surface Roughness}

Stone's parameters give a global measure of the roughness. For large terrain maps, a global measure is too general, and we will replace it by a local measure. To localize the terrain roughness, the input signal is filtered. One possible technique is to apply a window to the data to extract some subset of the samples. A rectangular window can be used to take a sequence of $N$ points and reduce it to a sequence of $M$ points - however this leads to undesirable high frequency components in the frequency spectrum (the Gibbs phenomenon). Because the roughness estimation uses all frequency components, we minimize the high frequency terms introduced by the filter. Of the three windows considered - Hamming, Hanning, and Blackman - the Blackman window introduces the smallest error in the $M, P$, and $K$ terms. The Blackman window is given by:

$$
b(n)=0.42-0.5 \cos \left(\frac{2 \pi n}{M}\right)+0.08 \cos \left(\frac{4 \pi n}{M}\right) n=0,1 \ldots, M
$$

\footnotetext{
${ }^{3}$ We assume a homogeneous surface, so a line fit rather than a plane fit is used.
} 
The goal is to transform the signal (to measure the intrinsic properties of the surface), localize it, and compute a roughness measurement. The specific steps are:

1. Take the original sampled signal $s(n)$ (of length $N$ ) and apply a rectangular window function $r(n)$ to it. This reduces the size of the signal from $N$ to $M$. Call the filtered signal $s_{r}(n)$ :

$$
s_{r}(n)=s(n) r(n)
$$

2. A least squares method fits a line to the signal $s_{r}(n)$. This line estimates the transformation $r$ that rotates and translates the intrinsic surface description $s_{i}(n)$ into $s_{r}(n)$ :

$$
s_{r}(n)=T s_{i}(n)
$$

3. Transform the signal $s_{r}(n)$ by $T$ determined in step (2) to remove the rotation and translation. More precisely:

$$
s_{i}(n)=T^{-1} s_{r}(n)
$$

4. Apply a Blackman window $b(n)$ of length $M$ to $s_{i}(n)$ to localize the measurement. Call the new signal $u(n)$ defined as:

$$
u(n)=s_{i}(n) b(n)
$$

5. Calculate the roughness parameters $M, P$, and $K$ for $u(n)$.

The steps enumerated find the $M, P, K$ values at a particular point. For a surface $s(n, m)$ this operation must be performed at each point.

\section{Experimental Results}

\subsection{Synthetic Terrain}

The extraction of the intrinsic roughness is illustrated in figure 2. The surface is a step function with added Gaussian noise.

The plots of $M$ and $P$ (figures 2(b) and 2(c)) show that the roughness is nearly the same, regardless of the amplitude. On a relative scale, the plot of $K$ (figure 2(d)) shows that the Gaussian noise component is essentially constant across the entire surface. However, the absolute scale is incorrect ( $K$ for Gaussian noise $\approx 0.98$, while the values for $K$ in the figure are between 0.8 and 0.85 ). The problem arises from the additional frequency terms produced in localization by the Blackman window. As the size of the Blackman window increases, the errors in $M, P, K$ decrease. Table 1 shows the values of $M, P, K$ for increasing window size. 


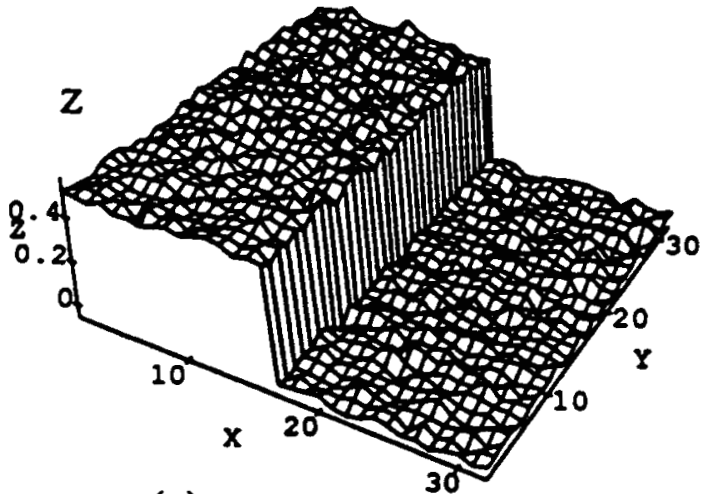

(a)

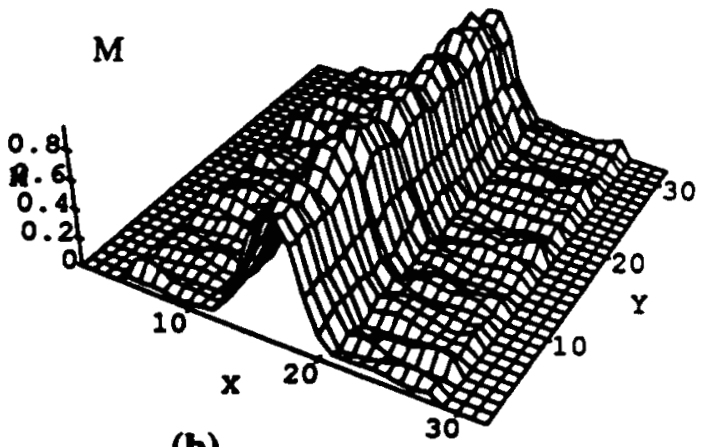

(b)

Step Function and $M$ Values

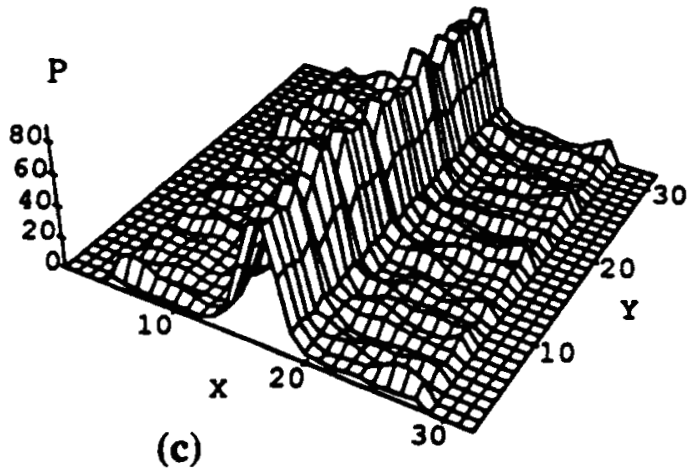

(c)

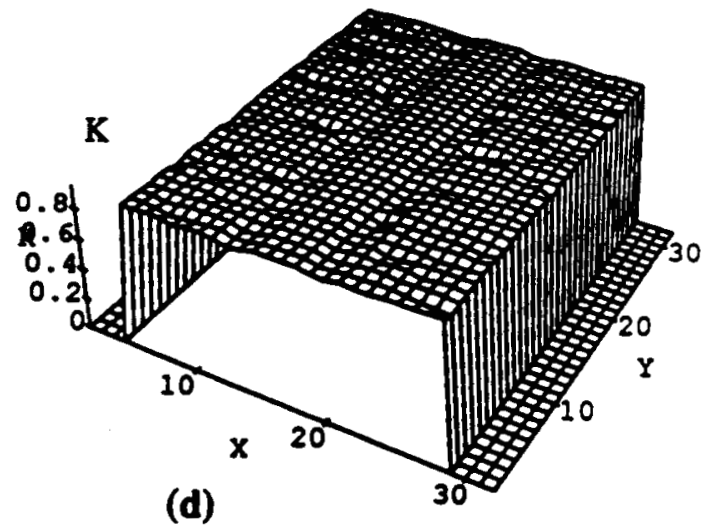

(d)

Figure 2: $\mathrm{P}$ and $\mathrm{K}$ Values for Step Function

\begin{tabular}{|r|r|r|r|}
\hline Size & $\mathbf{M}$ & $\mathbf{P}$ & $\mathbf{K}$ \\
4 & 2.7 & 148 & 0.93 \\
8 & 2.5 & 37 & 0.86 \\
12 & 2.4 & 16 & 0.77 \\
16 & 2.2 & 9.6 & 0.57 \\
\hline
\end{tabular}

Table 1: Effect of Window Size on $M, P, K$ 
The larger is the window size, the smaller is the error in the roughness measure. However, as the window size is increased, the edge effects become more pronounced, and useful data is lost. The $M, P, K$ values in figure 2 were computed for a window size of 8 . Because the Blackman window introduces error of 0.86 into $K$, the range of $K$ values of 0.8 to 0.85 in figure 2(d) are reasonable. (For unfiltered Gaussian noise, $K=K_{G} \approx 0.98$. The measured $K$ is $0.86 K_{G}$ ).

\subsection{Natural Terrain Data}

We applied this technique to natural terrain elevation data obtained at a nearby slag dump. This area consists of a $2 \mathrm{~m}$ road cut through small hills with heights up to $1 \mathrm{~m}$. The elevation map was created by merging a sequence of ERIM range sensor images taken by the CMU NavLab. It encompasses an area of $40 \mathrm{~m} \times 40 \mathrm{~m}$, has vertical resolution of $1 \mathrm{~cm}$, and $(x, y)$ grid spacing of $20 \mathrm{~cm}$.

Because of the computation time required to process an entire map, subregions of geological interest were chosen and analyzed. Figure $4(a)$ shows a $32 \times 32$ grid point region from the original map. (Figure 3 is a video image of the area). Figures 4 (b), (c) and (d) show the $M, P, K$ values at each point in the elevation map.

From the $M$ and $P$ plots, it is evident that two paths are relatively smooth - the left path ( $8 \leq x \leq 12)$ and the right path $(24 \leq x \leq 30)$. The left path corresponds to a road, the right path travels over a gently sloping ridge. Qualitatively, each path is acceptable in the sense that that it is less rough and more traversable than neighboring regions. The $K$ plot shows that the terrain has essentially the same distribution of a noise component over the entire surface, except at the elevation changes near surface discontinuities.

\section{Discussion}

We have presented a technique for estimating the local, intrinsic roughness of terrain. It relies on the statistical properties of the amplitude, slope, and correlation components of the terrain surface. Application to elevation maps of natural, irregular terrain shows that this method can identify regions of relative smoothness in the elevation map.

Future extensions include using this information to identify regions of traversability for mobile robots based on both the vehicle characteristics and terrain roughness. It may also be desirable to use all 2-D information (and replace the line fit estimate of the underlying surface with a plane fit) to obtain slope distributions in both $x$ and $y$ (although this increases the dimensionality of the problem). 


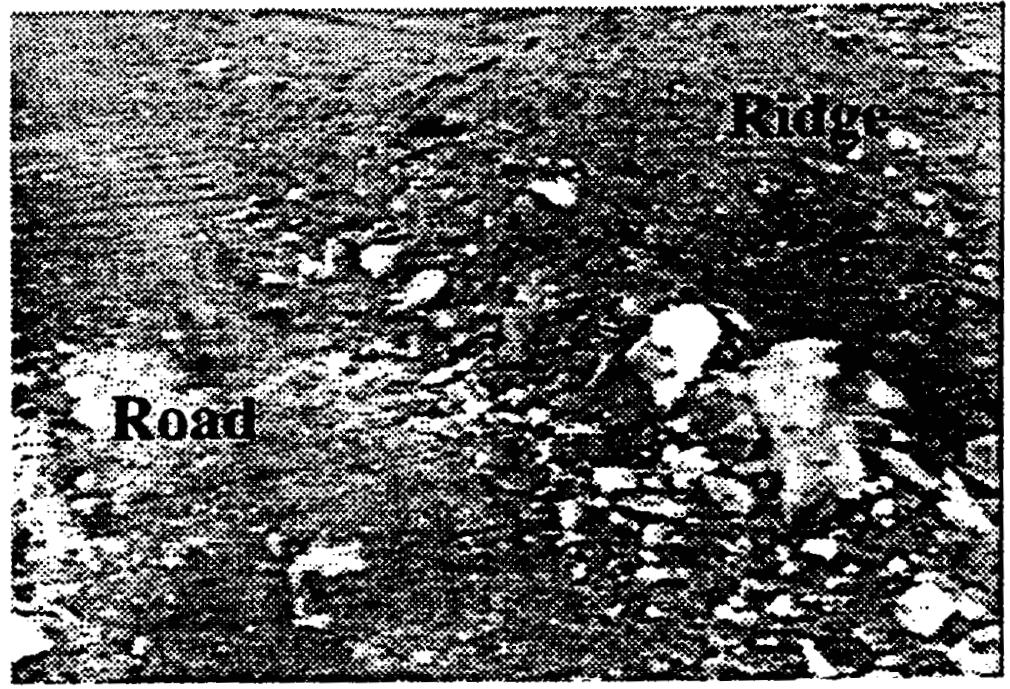

Figure 3: Road through Smail Hills

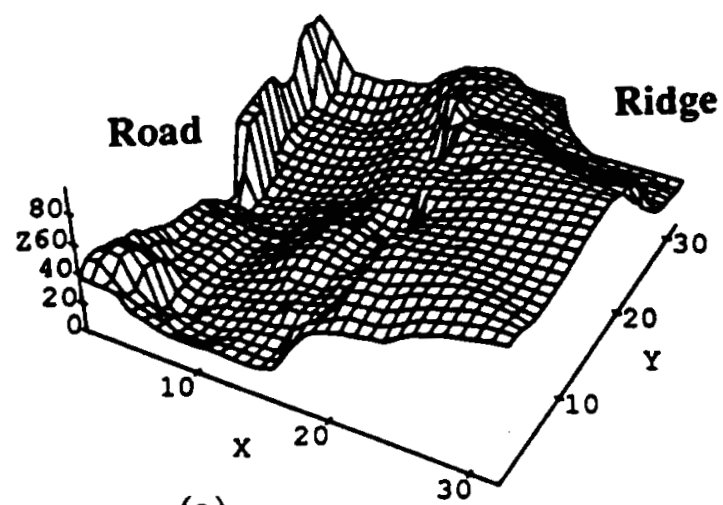

(a)

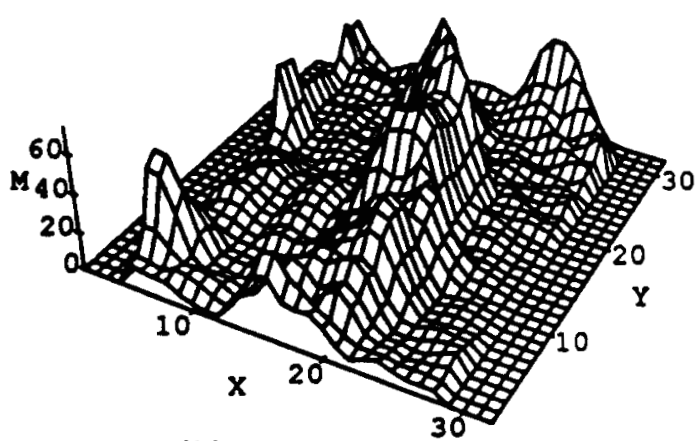

(b)

Terrain Elevation and $M$ Values

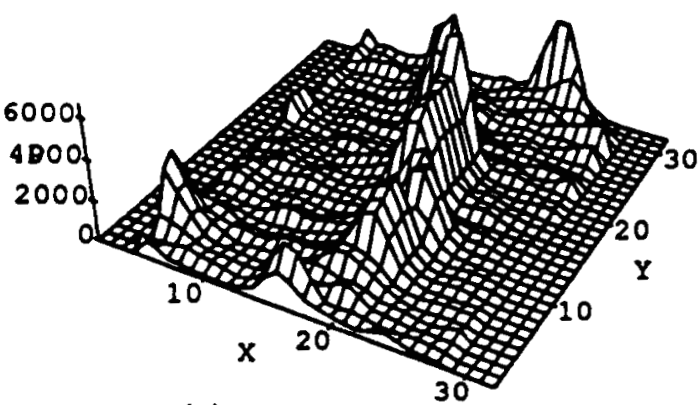

(c)

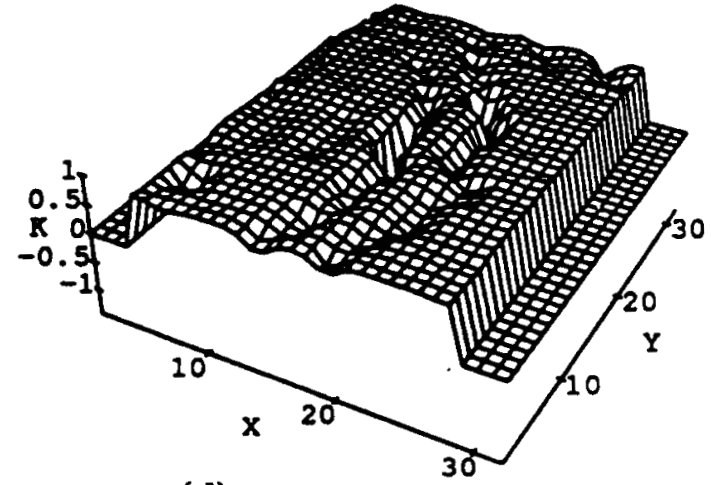

(d)

Figure 4: Terrain P and $\mathrm{K}$ Values for Slag Dump 


\section{References}

[1] J. Bares, M. Hebert, T. Kanade, E. Knotkov, T. Mitchell, R. Simmons, and W. Whittaker. Ambler: An Autonomous Rover for Planetary Explorations. IEEE Computer, 18-26, June 1989.

[2] M. Brock. Surface Roughness Analysis. Bruel and Kjaer Instruments, Inc., 1983.

[3] C. Caillas, M. Hebert, E. Krotkov, L.S. Kweon, and T. Kanade. Methods for Identifying Footfall Positions for a Legged Robot. In Proc. IEEE International Workshop on Intelligent Robots and Systems, pages 244-250, Tsukuba, Japan, September 1989.

[4] R.B. Daniels, L.A. Nelson, and E.E. Gamble. A Method of Characterizing Nearly Level Surfaces. Zeitschrifi fur Geomorphologie, 14(2):175-185, 1970.

[5] S. Franklin. Geomorphometric Processing of Digital Elevation Models. Computers and Geosciences, 13(6):603-609, 1987.

[6] M. Hebert, T. Kanade, E. Krotkov, and I.S. Kweon. Terrain Mapping for a Roving Planetary Explorer. In Proc. IEEE Robotics and Automation, pages 997-1002, Scottsdale, Arizona, May 1989.

[7] R. Stone and J. Dugundji. A Study of Microrelief - Its Mapping, Classification, and Quantification by Means of a Fourier Analysis. Engineering Geology, 1(2):89-187, 1965.

[8] K. J. Stout, C. Obray, and J. Jungles. Specification and Control of Surface Finish: Empiricism versus Dogmatism. Optical Engineering, 24:414-418, May/June 1985.

[9] D. J. Whitehouse. Typology of Manufactured Surfaces. Annals of the CIR.P., 19:417-431, June 1971.

[10] D. J. Whitehouse, T. King, and K. J. Stout. Surface Metrology: Its Relevance to Marginally Lubricated Bearing Performance. Surface Technology, 6:259-270, 1978.

[11] B. Wilcox and D. Gennery. A Mars Rover for the 1990's. Journal of the British Planetary Society, 40:484-488, 1987. 
